\title{
PERIODIC SOLUTIONS FOR THE GENERALIZED ANISOTROPIC LENNARD-JONES HAMILTONIAN
}

\author{
JAUME LLIBRE ${ }^{1}$ AND YIMING LONG ${ }^{2}$
}

\begin{abstract}
We characterize the circular periodic solutions of the generalized Lennard-Jones Hamiltonian system with two particles in $\mathbb{R}^{n}$, and we analyze what of these periodic solutions can be continued to periodic solutions of the anisotropic generalized Lennard-Jones Hamiltonian system.

We also characterize the periods of antiperiodic solutions of the generalized Lennard-Jones Hamiltonian system on $\mathbb{R}^{2 n}$, and prove the existences of $0<$ $\tau^{*} \leq \tau^{* *}$ such that this system possesses no $\tau / 2$-antiperiodic solution for all $\tau \in\left(0, \tau^{*}\right)$, at least one $\tau / 2$-antiperiodic solution when $\tau=\tau^{*}$, precisely $2^{n}$ families of $\tau / 2$-antiperiodic circular solutions when $\tau=\tau^{* *}$, and precisely $2^{n+1}$ families of $\tau / 2$-antiperiodic circular solutions when $\tau>\tau^{* *}$. Each of these circular solution families is of dimension $n-1$ module the $S^{1}$-action.
\end{abstract}

\section{Introduction AND STATEMENT OF THE MAIN RESUlts}

Frequently mathematical simple models are used in molecular dynamics and computational chemistry to describe the interaction between a pair of molecules or atoms, see for instance [5, 9].

One of the most used empirical potentials in molecular dynamics is the LennardJones potential, see [8], which models the interaction between two neutral atoms or molecules, under two different forces in the limit of small and large separation. These forces are: a repelling force at short distances (coming from overlapping electron orbitals, related to the Pauli's exclusion principle), and an attractive force at long distances (coming from the van der Waals force, or the dispersion force).

The Lennard-Jones potential

$$
V=a\left[\left(\frac{\sigma}{\left|r_{1}-r_{2}\right|}\right)^{6}-\left(\frac{\sigma}{\left|r_{1}-r_{2}\right|}\right)^{12}\right],
$$

where $a / 4$ is the depth of the potential energy, $\sigma$ is the finite distance at which the interparticle potential vanishes, $\left\|r_{1}-r_{2}\right\|$ is the distance between the two particles localized at the positions $r_{1}$ and $r_{2}$ in $\mathbb{R}^{n}$. The values of these parameters are chosen in order to reproduce experimental data, or deduced from accurate quantum chemistry computations; [3] is a good reference for these considerations.

We rescale the unit of length and the unit of mass in such a way that the constant $\sigma$ and $a$ become 1, then the Lennard-Jones potencial becomes

$$
V=\left(\frac{1}{\left|r_{1}-r_{2}\right|}\right)^{6}-\left(\frac{1}{\left|r_{1}-r_{2}\right|}\right)^{12} .
$$

1991 Mathematics Subject Classification. 70F10, 70H05, 34C23.

Key words and phrases. Lennard-Jones potential, circular periodic solutions, anisotropic Lennard-Jones potential. 
When one of the atoms or molecules is at the origin of coordinates and the position of the other atom or molecule is $x=\left(x_{1}, \ldots, x_{n}\right)$, then the Lennard-Jones Hamiltonian writes

$$
H_{L J}=\frac{1}{2} \sum_{k=1}^{n} p_{x_{k}}^{2}-\frac{1}{|x|^{6}}+\frac{1}{|x|^{12}}
$$

where $|x|=\sqrt{\sum_{k=1}^{n} x_{k}^{2}}$.

In the papers [2] and [13] the authors considered the so called generalized LennardJones Hamiltonian

$$
H_{g L J}=\frac{1}{2} \sum_{k=1}^{n} p_{x_{k}}^{2}-\frac{a}{|x|^{\alpha}}+\frac{b}{|x|^{\beta}},
$$

with $\beta>\alpha \geq 2, a>0$ and $b>0$. So the corresponding Hamiltonian system is

$$
\begin{aligned}
\dot{x}_{k} & =p_{x_{k}}, \\
\dot{p}_{x_{k}} & =\frac{a \alpha x_{k}}{|x|^{1+\alpha / 2}}-\frac{b \beta x_{k}}{|x|^{1+\beta / 2}},
\end{aligned}
$$

for $k=1, \ldots, n$.

In the coordinates $\left(x, p_{x}\right)$ the generalized Lennard-Jones potential is central, and consequently it is integrable with the independent first integrals given by the angular momentum $C=x \wedge p_{x}$, where $\wedge$ is the exterior product of the vectors $x$ and $p_{x}$. The norm of the angular momentum $C$ on a solution of the Hamiltonian system (2) is denoted by $c$, and of course it is also a first integral.

For stating our first result on the circular periodic solutions of the generalized Lennard-Jones Hamiltonian system we need some notation. We define

$$
\begin{aligned}
\rho_{1} & =\left(\frac{b \beta}{a \alpha}\right)^{\frac{1}{\beta-\alpha}}, \\
\rho_{2} & =\left(\frac{b \beta(\beta-2)}{a \alpha(\alpha-2)}\right)^{\frac{1}{\beta-\alpha}}, \\
\gamma & =\sqrt{\frac{a \alpha}{\rho_{2}^{\alpha-2}}-\frac{b \beta}{\rho_{2}^{\beta-2}}} .
\end{aligned}
$$

The next proposition characterizes the circular periodic solutions of the generalized Lennard-Jones Hamiltonian system.

Proposition 1. Assume $\beta>\alpha \geq 2, a>0$ and $b>0$. On the circular periodic solutions of the Lennard-Jones Hamiltonian system (2) the norm of the angular momentum $c$ takes values in the interval $[-\gamma, \gamma]$. In every 2 -dimensional plane through the origin of coordinates the following statement hold.

(a) For $c=-\gamma$ the Hamiltonian system (2) has only one retrograde circular periodic solution centered at the origin of coordinates of radius $\rho_{2}$. The period of this orbit is $2 \pi \rho_{2}^{2} / \gamma$. 
(b) For each $c \in(-\gamma, 0)$ the Hamiltonian system (2) has exactly two retrograde circular periodic solutions centered at the origin of coordinates, one with radius $r_{1}(c)$ in the interval $\left(\rho_{1}, \rho_{2}\right)$ of period

$$
\frac{2 \pi r_{1}(c)^{2}}{\sqrt{\frac{a \alpha}{r_{1}(c)^{\alpha-2}}-\frac{b \beta}{r_{1}(c)^{\beta-2}}}}
$$

this period tends to $2 \pi \rho_{2}^{2} / \gamma$ when $r_{1}(c) \nearrow \rho_{2}$ and tends to $\infty$ when $r_{1}(c) \searrow$ $\rho_{1}$; and the other periodic solution with radius $r_{2}(c)$ in the interval $\left(\rho_{2}, \infty\right)$ of period

$$
\frac{2 \pi r_{2}(c)^{2}}{\sqrt{\frac{a \alpha}{r_{2}(c)^{\alpha-2}}-\frac{b \beta}{r_{2}(c)^{\beta-2}}}}
$$

this period tends to $2 \pi \rho_{2}^{2} / \gamma$ when $r_{2}(c) \searrow \rho_{2}$ and tends to $\infty, 2 \pi / \sqrt{a \alpha}$ or 0 when $r_{2}(c) \nearrow \infty$ if $\alpha<6, \alpha=6$ or $\alpha>6$, respectively.

(c) For $c=0$ the Hamiltonian system (2) has a circle of equilibra centered at the origin of coordinates and of radius $\rho_{1}$.

(d) For each $c \in(0, \gamma)$ the Hamiltonian system (2) has exactly two direct circular periodic solutions centered at the origin of coordinates, one with radius $r_{1}(c)$ in the interval $\left(\rho_{1}, \rho_{2}\right)$, and the other with radius $r_{2}(c)$ in the interval $\left(\rho_{2}, \infty\right)$. The periods of these two orbits have the behavior described in statement $(b)$.

(e) For $c=\gamma$ the Hamiltonian system (2) has only one direct circular periodic solution centered at the origin of coordinates of radius $\rho_{2}$. The period of this orbit is the same than in statement $(a)$.

Proposition 1 is proved in section 2 .

Our first main goal is to characterize which of the circular periodic solutions described in Proposition 1 can be continued to the generalized anisotropic LennardJones Hamiltonian system defined by the Hamiltonian

$$
\begin{aligned}
H_{g a L J} & =\frac{1}{2} \sum_{k=1}^{n} p_{x_{k}}^{2}-\frac{a}{\left(\sum_{k=1}^{m} x_{k}^{2}+(1+\varepsilon) \sum_{k=m+1}^{n} x_{k}^{2}\right)^{\alpha / 2}}+\frac{b}{\left(\sum_{k=1}^{m} x_{k}^{2}+(1+\varepsilon) \sum_{k=m+1}^{n} x_{k}^{2}\right)^{\beta / 2}}, \\
& =H_{g L J}+\varepsilon\left(\frac{b \beta \sum_{k=m+1}^{n} x_{k}^{2}}{2|x|^{1+\beta / 2}}-\frac{a \alpha \sum_{k=m+1}^{n} x_{k}^{2}}{2|x|^{1+\alpha / 2}}\right)+O\left(\varepsilon^{2}\right) .
\end{aligned}
$$


for a given integer $m$ and a given $\varepsilon$ such that $1<m<n$ and $|\varepsilon|$ is sufficiently small. Therefore the corresponding Hamiltonian system is

(5)

$$
\begin{aligned}
\dot{x}_{k}= & p_{x_{k}}, \\
& \text { for } k=1, \ldots, n \\
\dot{p}_{x_{k}}= & \frac{a \alpha x_{k}}{\left(\sum_{k=1}^{m} x_{k}^{2}+(1+\varepsilon) \sum_{k=m+1}^{n} x_{k}^{2}\right)^{\alpha / 2}}-\frac{b \beta x_{k}}{\left(\sum_{k=1}^{m} x_{k}^{2}+(1+\varepsilon) \sum_{k=m+1}^{n} x_{k}^{2}\right)^{\beta / 2}},
\end{aligned}
$$

for $k=1, \ldots, m$

$$
\dot{p}_{x_{k}}=\frac{a \alpha(1+\varepsilon) x_{k}}{\left(\sum_{k=1}^{m} x_{k}^{2}+(1+\varepsilon) \sum_{k=m+1}^{n} x_{k}^{2}\right)^{\alpha / 2}}-\frac{b \beta(1+\varepsilon) x_{k}}{\left(\sum_{k=1}^{m} x_{k}^{2}+(1+\varepsilon) \sum_{k=m+1}^{n} x_{k}^{2}\right)^{\beta / 2}},
$$

for $k=m+1, \ldots, n$.

We define

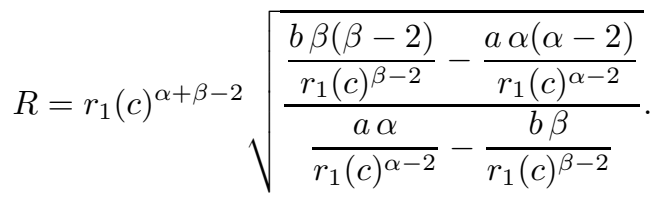

Our first main result is the following.

Theorem 2. Assume $\beta>\alpha \geq 2, a>0$ and $b>0$. The following statements hold.

(a) If $c \in(-\gamma, 0)$ and $R$ is not an integer, then at every 2 -dimensional plane $P$ through the origin of coordinates the retrograde circular orbit of radius $r_{1}(c)$ and angular momentum $c$ of the generalized Lennard-Jones Hamiltonian system (2) can be continued into the generalized anisotropic Lennard-Jones Hamiltonian system (5) for small values of $|\varepsilon|$.

(b) If $c \in(-\gamma, 0)$, then at every 2-dimensional plane $P$ through the origin of coordinates the retrograde circular orbit of radius $r_{2}(c)$ and angular momentum $c$ of the generalized Lennard-Jones Hamiltonian system (2) can be continued into the generalized anisotropic Lennard-Jones Hamiltonian system (5) for small values of $|\varepsilon|$.

(c) If $c \in(0, \gamma)$ and $R$ is not an integer, then at every 2-dimensional plane $P$ through the origin of coordinates the direct circular orbit of radius $r_{1}(c)$ and angular momentum $c$ of the generalized Lennard-Jones Hamiltonian system (2) can be continued into the generalized anisotropic Lennard-Jones Hamiltonian system (5) for small values of $|\varepsilon|$.

(d) If $c \in(0, \gamma)$, then at every 2-dimensional plane $P$ through the origin of coordinates the direct circular orbit of radius $r_{2}(c)$ and angular momentum $c$ of the generalized Lennard-Jones Hamiltonian system (2) can be continued into the generalized anisotropic Lennard-Jones Hamiltonian system (5) for small values of $|\varepsilon|$.

Theorem 2 is proved in section 4 . 
In what follows we shall characterize the periods of antiperiodic solutions of the Lennard-Jones Hamiltonian system on whether there exist such solutions.

For a given $\tau>0$ we study the $\tau$-periodic solutions of the Lennard--Jones Hamiltonian system (2), which now we rewrite into the form

$$
\ddot{x}(t)+U^{\prime}(x(t))=0,
$$

where $U \in C^{1}\left(\mathbb{R}^{2 n} \backslash\{0\}, \mathbb{R}\right)$ is defined by

$$
U(x)=-\frac{a}{|x|^{\alpha}}+\frac{b}{|x|^{\beta}}, \quad \forall x \in \mathbb{R}^{2 n},
$$

where we suppose $0<\alpha<\beta, a>0$ and $b \in \mathbb{R}$.

Firstly, for a given $\tau>0$ we plug the $\tau$-periodic circular motion $x=x(t)$ into the system (6) with the potential function $U=U(x)$ of $(7)$, and try to see which circular motion can become solution of (6).

Proposition 3. Let $U \in C^{1}\left(\mathbb{R}^{2} \backslash\{0\}, \mathbb{R}\right)$ be the potential function given by (7) with $0<\alpha<\beta, a>0$, and $b \in \mathbb{R}$. For $\tau>0$ and $r>0$ let

$$
\varphi_{\tau}(r) \equiv-\frac{4 \pi^{2}}{\tau^{2}} r^{\beta+2}+a \alpha r^{\beta-\alpha}-b \beta
$$

For $\tau>0$, let

$$
\begin{aligned}
& r_{0}(\tau)=\left(\frac{a \tau^{2} \alpha(\beta-\alpha)}{4 \pi^{2}(\beta+2)}\right)^{1 /(\alpha+2)}>0, \\
& \chi(\tau)=\varphi_{\tau}\left(r_{0}(\tau)\right)=\frac{a \alpha(\alpha+2)}{\beta+2}\left(\frac{a \tau^{2} \alpha(\beta-\alpha)}{4 \pi^{2}(\beta+2)}\right)^{(\beta-\alpha) /(\alpha+2)}-b \beta,
\end{aligned}
$$

where $r=r_{0}(\tau)$ is the only critical point of $\varphi_{\tau}(r)$ on $r>0$. Then the following holds.

(i) For $\tau>0$ satisfying $\chi(\tau)>0$, system (6) possesses precisely $4 \tau / 2-$ antiperiodic circular solution $S^{1}$-orbits centered at the origin given by

$$
x_{ \pm, r_{i}(\tau), t_{i}}(t)=\left(r_{i}(\tau) \cos \frac{2 \pi\left(t-t_{i}\right)}{\tau}, \pm r_{i}(\tau) \sin \frac{2 \pi\left(t-t_{i}\right)}{\tau}\right)
$$

where $r_{1}(\tau)$ and $r_{2}(\tau)$ are two positive roots of $\varphi_{\tau}(r)$ satisfying $0<r_{1}(\tau)<$ $r_{0}(\tau)<r_{2}(\tau)<+\infty$, and $t_{i} \in \mathbb{R}$ for $i=1,2$.

(ii) For $\tau>0$ satisfying $\chi(\tau)=0$, system (6) possesses precisely $2 \tau / 2-$ antiperiodic circular solution $S^{1}$-orbits centered at the origin given by

$$
x_{ \pm, r_{0}(\tau), t_{0}}(t)=\left(r_{0}(\tau) \cos \frac{2 \pi\left(t-t_{0}\right)}{\tau}, \pm r_{0}(\tau) \sin \frac{2 \pi\left(t-t_{0}\right)}{\tau}\right)
$$

where $t_{0} \in \mathbb{R}$.

(iii) For $\tau>0$ satisfying $\chi(\tau)<0$, system (6) possesses no $\tau / 2$-antiperiodic circular solutions centered at the origin.

Proposition 3 is proved in Section 5 below.

By a similar proof which is left to the readers, we have the following result for the higher dimensional case.

Proposition 4. Let $U \in C^{1}\left(\mathbb{R}^{2 n} \backslash\{0\}, \mathbb{R}\right)$ be the potential function given by $(7)$ with $0<\alpha<\beta, a>0$, and $b \in \mathbb{R}$. For $\tau>0$ and $r>0$ define $\varphi_{\tau}(r), r_{0}(\tau)$ and $\chi(\tau)$ by (8), (9) and (10) respectively. Then the following hold. 
(i) For $\tau>0$ satisfying $\chi(\tau)>0$, system (6) possesses precisely the following $2^{n+1}$ families of $\tau / 2$-antiperiodic circular solution $S^{1}$-orbits centered at the origin given by $x(t)=\left(x_{1}(t), \ldots, x_{n}(t)\right)$, where

$$
x_{i}(t)=\left(R_{i} \cos \frac{2 \pi\left(t-t_{i}\right)}{\tau}, \pm R_{i} \sin \frac{2 \pi\left(t-t_{0}\right)}{\tau}\right)
$$

with $t_{i} \in \mathbb{R}, R_{i} \in[0, R]$ for $1 \leq i \leq n$ satisfying

$$
\sum_{i=1}^{n} R_{i}^{2}=r_{j}(\tau)^{2}
$$

and $r_{j}(\tau)>0$ with $j=1$ or 2 is one of the two positive roots $0<r_{1}(\tau)<$ $r_{2}(\tau)$ of the function $\varphi_{\tau}(r)$. These families are determined by $r_{1}(\tau)$ and $r_{2}(\tau)$ as well as $2^{n}$ choices of orientations of the 2-dimensional circular branch orbits respectively. Each of these families is of dimension $n-1$ in the parameters $R_{1}, \ldots, R_{n}$ satisfying (14) module the $S^{1}$-action.

(ii) For $\tau>0$ satisfying $\chi(\tau)=0$, system (6) possesses precisely the following $2^{n}$ families of $\tau / 2$-antiperiodic circular solution $S^{1}$-orbits centered at the origin given by (13) and (14) with $R>0$ being the only positive root $r_{0}(\tau)$ of the function $\varphi_{\tau}(r)$. These families are determined by $r_{0}(\tau)$ and the $2^{n}$ choices of orientations of the 2 -dimensional circular branch orbits respectively. Each of these families is of dimension $n-1$ in the parameters $R_{1}, \ldots, R_{n}$ satisfying (14) module the $S^{1}$-action.

(iii) For $\tau>0$ satisfying $\chi(\tau)<0$, system (6) possesses no $\tau / 2$-antiperiodic circular solutions centered at the origin.

Because $U$ satisfies $U(-x)=U(x)$ for any $x \in \mathbb{R}^{2 n} \backslash\{0\}$, we can look for $\tau / 2$ antiperiodic solutions of (6), i.e. those solutions $x$ satisfying

$$
x\left(t+\frac{\tau}{2}\right)=-x(t), \quad \forall t \in \mathbb{R} .
$$

Note that circular solutions of (6) found by Proposition 3 are all $\tau / 2$-antiperiodic solutions. It is well known that $\tau / 2$-antiperiodic solutions of (6) are critical points of the functional

defined on the space

$$
f_{\tau}(x)=\int_{0}^{\tau}\left(\frac{1}{2}|\dot{x}|^{2}-U(x)\right) d t
$$

$$
X_{\tau}=\left\{x \in W^{1,2}\left(S_{\tau}, \mathbb{R}^{2 n}\right) \mid x\left(t+\frac{\tau}{2}\right)=-x(t), \forall t \in \mathbb{R}\right\},
$$

where $S_{\tau}=\mathbb{R} /(\tau \mathbb{Z})$.

Motivated by the method of [11], we obtain our second main result below. Here this theorem characterizes the period $\tau>0$ for which (6) possesses no, at least one or more $\tau / 2$-antiperiodic solutions.

Theorem 5. For any given constants $2<\alpha<\beta, a>0$, and $b>0$, let $\tau^{* *}>0$ be the unique positive root of the function $\chi(\tau)$ of $(10)$. Then there exists a positive real number $\tau^{*} \in\left(0, \tau^{* *}\right]$ such that the system (6) possesses no $\tau / 2$-antiperiodic solutions when $0<\tau<\tau^{*}$, at least one $\tau / 2$-antiperiodic solution when $\tau=\tau^{*}$, precisely $2^{n}$ families of $\tau / 2$-antiperiodic circular solutions when $\tau=\tau^{* *}$, and precisely $2^{n+1}$ families of $\tau / 2$-antiperiodic circular solutions when $\tau>\tau^{* *}$. Each of these circular solution families is of dimension $n-1$ module the $S^{1}$-action. 


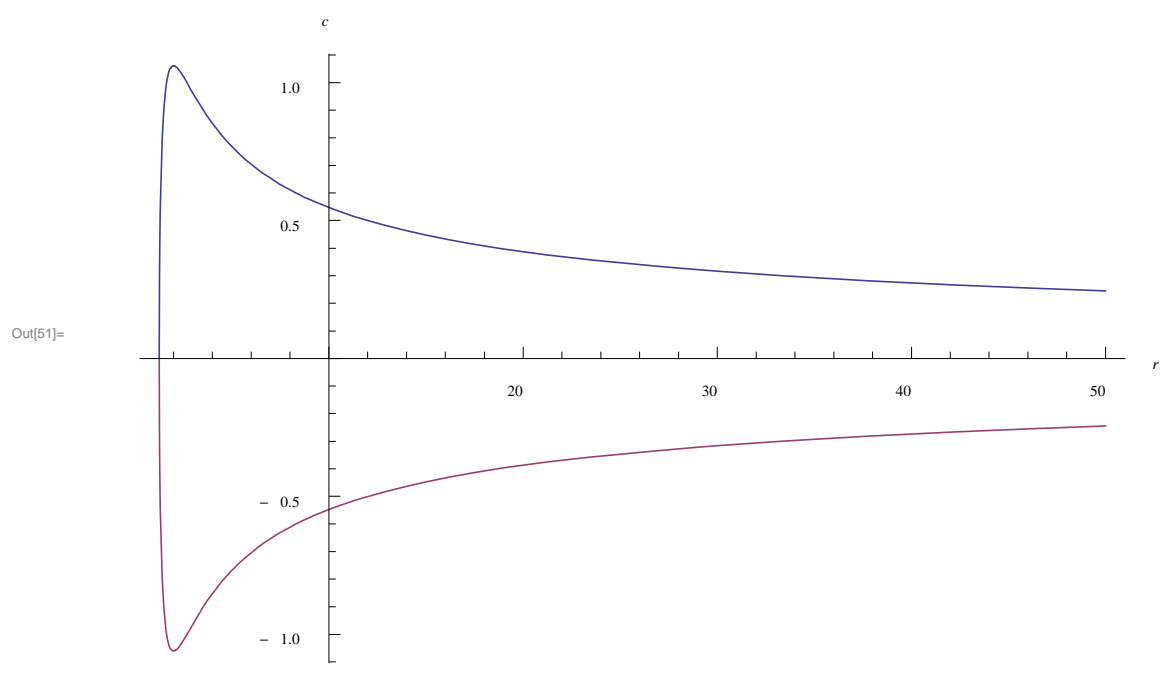

FiguRE 1. The graphic of the function $c(r)$.

Theorem 5 is proved in Section 6 below.

See $[1,4,14]$ and the references therein for other works on the periodic orbits of the Lennard-Jones potential, or related with these periodic orbits.

\section{Proof of Proposition 1}

Since the notion of angular momentum is defined in any dimension by using the exterior product in $\mathbb{R}^{n}$, one would guess that central force problems in any dimension are completely integrable, as it is well known for $n=3$. This was proved explicitly in [7], by constructing $n$ first integrals independent and involution: the energy and some combinations of the angular momentum components. It is shown that the motion of these central problems are always reduced to a 2-dimensional plane through the origin of coordinates.

The Lennard-Jones Hamiltonian (1), restricted to a 2-dimensional plane $P$ through the origin of coordinates with initial position and momenta in $P$, in polar coordinates in $P$ becomes

$$
H=\frac{1}{2}\left(\frac{\Theta^{2}}{r^{2}}+R^{2}\right)-\frac{a}{r^{\alpha}}+\frac{b}{r^{\beta}},
$$

where $\Theta$ is the norm of the angular momentum restricted to $P$ in polar coordinates. Therefore its corresponding Hamiltonian system writes

$$
\begin{aligned}
\dot{r} & =R, \\
\dot{\theta} & =\frac{\Theta}{r^{2}}, \\
\dot{R} & =\frac{\Theta^{2}}{r^{3}}-\frac{a \alpha}{r^{1+\alpha}}+\frac{b \beta}{r^{1+\beta}}, \\
\dot{\Theta} & =0 .
\end{aligned}
$$


We fix the value of $\Theta=c$. On a circular periodic solution in $P$ we have $\dot{r}=R=0$. Therefore

$$
\dot{R}=\frac{c^{2}}{r^{3}}-\frac{a \alpha}{r^{1+\alpha}}+\frac{b \beta}{r^{1+\beta}}=0 .
$$

Hence the value of the angular momentum over the circular periodic solution of radius $r$ in $P$ is

$$
c(r)= \pm \sqrt{\frac{a \alpha}{r^{\alpha-2}}-\frac{b \beta}{r^{\beta-2}}} .
$$

Therefore the radius of a circular periodic orbit must satisfy $r \geq \rho_{1}$. See the graphic of the function $c(r)$ in Figure 1.

The maximum and the minimum of the function $c(r)$ takes place when $r=\rho_{2}$ and $c\left(\rho_{2}\right)= \pm \gamma$. So the value of the angular momentum on the circular periodic solutions in $P$ run in the interval $[-\gamma, \gamma]$, as it is stated in Proposition 1. Now the statements of Proposition 1 follows easily from Figure 1.

\section{BASIC RESUlts ON THE CONTINUATION OF PERIODIC SOLUTIONS}

We deal with autonomous differential systems

$$
\dot{x}=f(x ; \varepsilon),
$$

where $f: U \times\left(-\varepsilon_{0}, \varepsilon_{0}\right) \rightarrow \mathbb{R}^{m}$ is $C^{2}, U$ is an open subset of $\mathbb{R}^{m}, x \in U,\left(-\varepsilon_{0}, \varepsilon_{0}\right)$ with $\varepsilon_{0}>0$ is an interval where the parameter $\varepsilon$ takes values, and as usual the dot denotes the derivative with respect to the time $t$. We denote its general solution as $\phi\left(t, x_{0} ; \varepsilon\right)$ satisfying $\phi\left(0, x_{0} ; \varepsilon\right)=x_{0} \in U$, here $t$ belongs to the maximal interval of definition of this solution.

A solution $\phi\left(t, x_{0} ; \varepsilon\right)$ is $T$-periodic with $T>0$ if and only if $\phi\left(T, x_{0} ; \varepsilon\right)=x_{0}$ and $\phi\left(t, x_{0} ; \varepsilon\right) \neq x_{0}$ for $t \in(0, T)$. The periodic orbit associated to the periodic solution $\phi\left(t, x_{0} ; \varepsilon\right)$ is $\gamma=\left\{\phi\left(t, x_{0} ; \varepsilon\right), t \in[0, T]\right\}$.

Consider the $T$-periodic solution $\phi\left(t, x_{0} ; 0\right)$. A continuation of this periodic solution is a pair of smooth functions, $u(\varepsilon), \tau(\varepsilon)$, defined for $|\varepsilon|$ sufficiently small such that $u(0)=x_{0}, \tau(0)=T$ and $\phi(t, u(\varepsilon) ; \varepsilon)$ is $\tau(\varepsilon)$-periodic. One also says that the periodic solution can be continued. This means that the solution persists when the parameter $\varepsilon$ varies, and the periodic solution does not change very much with the parameter.

The variational equation associated to the $T$-periodic solution $\phi\left(t, x_{0} ; \varepsilon\right)$ is

$$
\dot{M}=\left(\left.f_{x}(x ; \varepsilon)\right|_{x=\phi\left(t, x_{0} ; \varepsilon\right)}\right) M,
$$

where $M$ is a $m \times m$ matrix. Note that the matrix $f_{x}(x ; \varepsilon)$ is the Jacobian matrix of the vector field $f(x ; \varepsilon)$.

The monodromy matrix associated to the $T$-periodic solution $\phi\left(t, x_{0} ; \varepsilon\right)$ is the solution $M\left(T, x_{0} ; \varepsilon\right)$ of (18) satisfying that $M\left(0, x_{0} ; \varepsilon\right)$ is the identity matrix of $\mathbb{R}^{m}$. The eigenvalues of the monodromy matrix associated to the periodic solution $\phi\left(t, x_{0} ; \varepsilon\right)$ are called the multipliers of the periodic orbit.

Let $\phi\left(t, x_{0} ; \varepsilon\right)$ be a $T$-periodic orbit of the $C^{2}$ differential system (17). The eigenvector tangent to the periodic orbit has associated an eigenvalue equal to 1 . So the periodic orbit has at least one multiplier equal to 1 , for more details see for instance Proposition 1 in [10]. 
Let $F: U \rightarrow \mathbb{R}$ be a locally non-constant function of class $C^{1}$ such that

$$
\nabla F(x) \cdot f(x ; \varepsilon)=0 .
$$

Then $F$ is called a first integral of system (17), because $F$ is constant on the solutions of this system. Here the dot - indicates the usual inner product of $\mathbb{R}^{m}$, and the gradient of $F$ is defined as

$$
\nabla F(x)=\left(\frac{\partial F}{\partial x_{1}}, \ldots, \frac{\partial F}{\partial x_{n}}\right)
$$

We say that $k$ first integrals $F_{j}: U \rightarrow \mathbb{R}$ for $j=1, \ldots, k$ are linearly independent if their gradients are independent in all the points of $U$ except perhaps in a set of Lebesgue measure zero.

Let $F_{j}: U \rightarrow \mathbb{R}$ a first integral for $j=1, \ldots, k$ with $k<m$. Assume that $F_{1}, \ldots, F_{k}$ are linearly independent in $U$. Let $\gamma$ be a $T$-periodic orbit of the vector field $f(x ; \varepsilon)$ such that at every point $x \in \gamma$ the vectors $\nabla F_{1}(x), \ldots, \nabla F_{k}(x)$ and $f(x ; \varepsilon)$ are linearly independent. Then 1 is a multiplier of the periodic orbit $\gamma$ with multiplicity at least $k+1$, see for instance Theorem 2 of [10].

If the differential system (17) has $k$ independent first integrals, we say that a periodic solution $\phi\left(t, x_{0} ; \varepsilon\right)$ is non-degenerate if 1 is an eigenvalue of the monodromy matrix $M\left(T, x_{0} ; \varepsilon\right)$ with multiplicity $k+1$. The following result goes back to Poincaré, for a proof see for instance the proof of Proposition 9.1.1 of [12].

Proposition 6. A non-degenerate periodic solution of a differential system (17) with $\varepsilon=0$ and $k$ independent first integrals can be continued to differential systems (17) with $|\varepsilon|$ sufficiently small.

\section{Proof of Theorem 2}

We shall work in a fixed 2 -dimensional plane $P$ through the origin of coordinates in the space of positions. In fact, from [7] we know that we can find $2 n-3$ independent first integrals, such that $2 n-4$ fix the motion on the plane $P$, and the additional first integral is the restriction of the Hamiltonian of the system to the invariant plane $P$. From section 3 it follows that a circular periodic solution in the plane $P$ is non-degenerate if it has $2 n-2$ multipliers equal to 1 , and the remainder two are different from 1 . Since we shall work with the differential system (17) restricted to the invariant position plane $P$, in order to see that a circular periodic solution contained in $P$ is non-degenerate it is sufficient to prove that their multipliers are 1 with multiplicity two, and two other multipliers different from 1 .

For $c \in(-\gamma, 0)$ we consider the circular periodic solution of radius $r_{1}(c) \in\left(\rho_{1}, \rho_{2}\right)$ in $P$, see statement (b) of Proposition 1. On this periodic solution $R=0$, and from (15) its angular velocity is $\dot{\theta}=\omega=c / r_{1}(c)^{2}$. Therefore its period is

$$
T(c)=\frac{2 \pi}{\omega}=\frac{2 \pi r_{1}(c)^{2}}{|c|}
$$

Note that the period is the necessary time in order that $\theta$ increases or decreases by $2 \pi$. 
The Jacobian matrix of the Hamiltonian vector field corresponding to the Hamiltonian system $(15)$ is

$$
\left(\begin{array}{cccc}
0 & 0 & 1 & 0 \\
-\frac{2 \Theta}{r^{3}} & 0 & 0 & \frac{1}{r^{2}} \\
\frac{a \alpha(\alpha+1)}{r^{\alpha+2}}-\frac{b \beta(\beta+1)}{r^{\beta+2}}-\frac{3 \Theta^{2}}{r^{4}} & 0 & 0 & \frac{2 \Theta}{r^{3}} \\
0 & 0 & 0 & 0
\end{array}\right)
$$

When we evaluate this matrix on the circular periodic solution of radius $r_{1}(c)$ and $c \in(-\gamma, 0)$ with

$$
\Theta=c=-\sqrt{\frac{a \alpha}{r_{1}(c)^{\alpha-2}}-\frac{b \beta}{r_{1}(c)^{\beta-2}}},
$$

recall (16), we obtain the matrix

$$
A=\left(\begin{array}{cccc}
0 & 0 & 1 & 0 \\
-2 \sqrt{\frac{a \alpha}{r_{1}(c)^{\alpha+4}}-\frac{b \beta}{r_{1}(c)^{\beta+4}}} & 0 & 0 & \frac{1}{r_{1}(c)^{2}} \\
\frac{a \alpha(\alpha-2)}{r_{1}(c)^{\alpha+2}}-\frac{b \beta(\beta-2)}{r_{1}(c)^{\beta+2}} & 0 & 0 & 2 \sqrt{\frac{a \alpha}{r_{1}(c)^{\alpha+4}}-\frac{b \beta}{r_{1}(c)^{\beta+4}}} \\
0 & 0 & 0 & 0
\end{array}\right)
$$

Now the variational equation (18) becomes

$$
\dot{M}=A M
$$

where $M$ is a $4 \times 4$ matrix, and the solution $M(t)$ of this differential equation such that $M(0)$ is the identity matrix of $\mathbb{R}^{4}$, evaluated at the period (19) of the circular periodic orbit of radius $r_{1}(c)$ is

$$
\left(\begin{array}{cccc}
C & 0 & \frac{r^{2} S}{\sqrt{A}} & \frac{2 r_{1} \sqrt{B}(C-1)}{A} \\
-\frac{2}{r} \sqrt{\frac{B}{A}} S & 1 & -\frac{2 r_{1} \sqrt{B}(C-1)}{A} & D \\
\frac{\sqrt{A} S}{r^{2}} & 0 & C & \frac{2 \sqrt{B} S}{\sqrt{A} r} \\
0 & 0 & 0 & 1
\end{array}\right)
$$


where $r_{1}=r_{1}(c)$, and

$$
\begin{aligned}
& A=\frac{a \alpha(\alpha-2)}{r_{1}^{\alpha-2}}-\frac{b \beta(\beta-2)}{r_{1}^{\beta-2}}, \\
& B=\frac{a \alpha}{r_{1}^{\alpha-2}}-\frac{b \beta}{r_{1}^{\beta-2}}, \\
& C=\cosh \left(2 \pi \sqrt{\frac{A}{B}}\right) \\
& S=\sinh \left(2 \pi \sqrt{\frac{A}{B}}\right), \\
& D=\frac{2 \pi\left(a \alpha(\alpha+2) r_{1}^{\beta}-b \beta(\beta+2) r_{1}^{\alpha}\right)}{r_{1}^{\alpha+\beta-2} A \sqrt{B}}-4 r_{1}^{2 \alpha+2 \beta+2} B \sqrt{A} S .
\end{aligned}
$$

Of course, by definition this last matrix is the monodromy matrix of the circular periodic solution of radius $r_{1}(c)$. Its eigenvalues are the multipliers of this periodic solution, namely

$$
1, \quad 1, \quad \pm e^{-2 \pi \sqrt{\frac{A}{B}}} .
$$

Since $r_{1} \in\left(\rho_{1}, \rho_{2}\right)$ we have that $A B<0$, and consequently

$$
e^{-2 \pi i \sqrt{-\frac{A}{B}}} \neq 1
$$

if and only if

$$
\sqrt{-\frac{A}{B}} \text { is not an integer. }
$$

Of course as usual $i=\sqrt{-1}$. Now applying Proposition 6 it follows statement (a) of Theorem 2.

For $c \in(-\gamma, 0)$ we consider the circular periodic solution of radius $r_{2}(c) \in$ $\left(\rho_{2}, \infty\right)$, see statement (c) of Proposition 1. Then doing the same kind of computations than for the circular periodic solution of radius $r_{1}(c)$ we obtain for the circular periodic solution of radius $r_{2}(c)$ the same multipliers $(20)$ but with $r_{2}(c)$ instead of $r_{1}(c)$, Since $r_{2}(c) \in\left(\rho_{2}, \infty\right)$ we have that $A B>0$, then the multipliers

$$
\pm e^{2 \pi \sqrt{\frac{A}{B}}}
$$

never can be 1 , and consequently by Proposition 6 , statement (b) of Theorem 2 follows.

Finally, the proofs of statements (c) and (d) of Theorem 2 are the same than the proofs of statements (a) and (b) of of Theorem 2 . 
5. Finding $\tau / 2$-ANTiPERIOdic CirCular solutions of SyStem (6)

Proof of Proposition 3. By definition (7) of $U$, we obtain

$$
U^{\prime}(x)=\frac{a \alpha}{|x|^{\alpha+2}} x-\frac{b \beta}{|x|^{\beta+2}} x .
$$

Let $x_{ \pm, r}(t)=\left(r \cos \frac{2 \pi t}{\tau}, \pm r \sin \frac{2 \pi t}{\tau}\right)$ for some $r>0$ to be determined later. Then we obtain

$$
\ddot{x}_{ \pm, r}(t)=-\frac{4 \pi^{2}}{\tau^{2}} x_{ \pm, r}(t)
$$

Thus we have

(23) $\ddot{x}(t)+U^{\prime}(x(t))=\left[-\frac{4 \pi^{2}}{\tau^{2}}+a \alpha r^{-(\alpha+2)}-b \beta r^{-(\beta+2)}\right] x(t)=r^{-(\beta+2)} \varphi_{\tau}(r) x(t)$.

That is, $x$ is a solution of (6) if and only if $r>0$ is a root of $\varphi_{\tau}(r)$.

Note that $\varphi_{\tau}(r)$ is continuous for $r \in[0,+\infty)$ and $C^{\infty}$ for $r>0$. By direct computations, we obtain

$$
\begin{aligned}
\varphi_{\tau}(r) & =r^{\beta-\alpha}\left(a \alpha-\frac{4 \pi^{2}}{\tau^{2}} r^{\alpha+2}\right)-b \beta \\
\varphi_{\tau}^{\prime}(r) & =a \alpha(\beta-\alpha) r^{\beta-\alpha-1}-\frac{4 \pi^{2}(\beta+2)}{\tau^{2}} r^{\beta+1} \\
& =r^{\beta-\alpha-1}\left(a \alpha(\beta-\alpha)-\frac{4 \pi^{2}(\beta+2)}{\tau^{2}} r^{\alpha+2}\right), \\
\varphi_{\tau}^{\prime \prime}(r) & =a \alpha(\beta-\alpha)(\beta-\alpha-1) r^{\beta-\alpha-2}-\frac{4 \pi^{2}(\beta+2)(\beta+1)}{\tau^{2}} r^{\beta} \\
& =r^{\beta-\alpha-2}\left(a \alpha(\beta-\alpha)(\beta-\alpha-1)-\frac{4 \pi^{2}(\beta+2)(\beta+1)}{\tau^{2}} r^{\alpha+2}\right) .
\end{aligned}
$$

Then $\varphi_{\tau}^{\prime}(r)=0$ for $r>0$ yields that $\varphi_{\tau}(r)$ possesses precisely one critical point $r=r_{0}=r_{0}(\tau)$,

At this point we have

$$
r_{0}=\left(\frac{a \tau^{2} \alpha(\beta-\alpha)}{4 \pi^{2}(\beta+2)}\right)^{1 /(\alpha+2)}>0 .
$$

$$
\begin{aligned}
\varphi_{\tau}^{\prime \prime}\left(r_{0}\right) & =r_{0}^{\beta-\alpha-2}\left(a \alpha(\beta-\alpha)(\beta-\alpha-1)-\frac{4 \pi^{2}(\beta+2)(\beta+1)}{\tau^{2}} r_{0}^{\alpha+2}\right) \\
& =-a \alpha(\beta-\alpha)(\alpha+2) r_{0}^{\beta-\alpha-2}<0 .
\end{aligned}
$$

Thus $r=r_{0}$ is a maximal point of $\varphi_{\tau}(r)$, at which we have

Note that we have

$$
\begin{aligned}
\chi(\tau) \equiv \varphi_{\tau}\left(r_{0}\right) & =r_{0}^{\beta-\alpha}\left(a \alpha-\frac{4 \pi^{2}}{\tau^{2}} \frac{a \tau^{2} \alpha(\beta-\alpha)}{4 \pi^{2}(\beta+2)}\right)-b \beta \\
& =\frac{a \alpha(\alpha+2)}{\beta+2}\left(\frac{a \tau^{2} \alpha(\beta-\alpha)}{4 \pi^{2}(\beta+2)}\right)^{(\beta-\alpha) /(\alpha+2)}-b \beta .
\end{aligned}
$$

$$
\lim _{r \rightarrow 0^{+}} \varphi_{\tau}(r)=-b \beta \text { and } \lim _{r \rightarrow+\infty} \varphi_{\tau}(r)=-\infty .
$$

Therefore under the assumptions of statement (i) of Proposition 3, i.e., $\tau>0$ satisfying $\chi(\tau)>0$, the function $\varphi_{\tau}(r)$ for $r>0$ possesses precisely two positive 
roots, which we denote by $r_{1}(\tau)$ and $r_{2}(\tau)$ satisfying $0<r_{1}(\tau)<r_{0}(\tau)<r_{2}(\tau)<$ $+\infty$. Considering orientation, they yield four $\tau / 2$-antiperiodic circular solution $S^{1}$-orbits centered at the origin

$$
x_{ \pm, r_{i}(\tau), t_{i}}(t)=\left(r_{i}(\tau) \cos \frac{2 \pi\left(t-t_{i}\right)}{\tau}, \pm r_{i}(\tau) \sin \frac{2 \pi\left(t-t_{i}\right)}{\tau}\right)
$$

for $t_{i} \in \mathbb{R}$ with $i=1,2$.

Under the assumptions of statement (ii) of Proposition 3, i.e., $\tau>0$ satisfying $\chi(\tau)=0$, the function $\varphi_{\tau}(r)$ for $r>0$ possesses precisely only one positive root $r=r_{0}(\tau)$. Thus considering orientation, they yield two $\tau / 2$-antiperiodic circular solution $S^{1}$-orbits centered at the origin given by $(24)$ with $i=0$ and $r_{i}(\tau)=r_{0}(\tau)$ and $t_{0} \in \mathbb{R}$.

Under the assumptions of statement (iii) of Proposition 3, i.e., $\tau>0$ satisfying $\chi(\tau)<0$, the function $\varphi_{\tau}(r)$ for $r>0$ possesses no positive roots, and therefore system (6) possesses no $\tau / 2$-antiperiodic circular solution $S^{1}$-orbits centered at the origin for all $\tau>0$.

This completes the proof of Proposition 3.

Next we give an example for the weak-force case $0<\alpha=1<\beta=2$, which does not satisfy specially the condition $2<\alpha<\beta$ of Theorem 5 .

Example 1. Let $\alpha=1, \beta=2, a=b=1$ in (7). Then for $\tau>0$, we have

$$
r_{0}(\tau)=\left(\frac{\tau^{2}}{16 \pi^{2}}\right)^{1 / 3}, \quad \chi(\tau)=\frac{3}{4}\left(\frac{\tau^{2}}{16 \pi^{2}}\right)^{1 / 3}-2 .
$$

Then the three cases of Proposition 3 hold, and system (6) possesses $\tau / 2$-antiperiodic circular solutions when $\tau>0$ is large enough.

\section{Proof of Theorem 5}

In order to prove Theorem 5 we need the following two inequalities given in the next two lemmas.

Lemma 7 (Wirtinger's inequality, cf. Theorem 258 of [6]). For real numbers $a<b$, let $f \in W^{1,2}([a, b], \mathbb{R})$ satisfying $f(a)=f(b)$ and $\int_{a}^{b} f(t) d t=0$. Then

$$
\left(\frac{2 \pi}{b-a}\right)^{2} \int_{a}^{b}[f(t)]^{2} d t \leq \int_{a}^{b}\left[f^{\prime}(t)\right]^{2} d t
$$

and the equality holds if and only if

$$
f(t)=c_{1} \cos \frac{2 \pi(t-a)}{b-a}+c_{2} \sin \frac{2 \pi(t-a)}{b-a},
$$

for some constant $c_{1}$ and $c_{2} \in \mathbb{R}$.

Lemma 8 (Jensen's inequality, cf. Theorem 204 of [6]). For real numbers $a<b$, let $\phi=\phi(t)$ satisfying $\phi^{\prime \prime}(t)>0$ and be finite for all $t \in(a, b)$, and $f$ and $p$ be integrable on $[a, b]$ and satisfying

$$
0<\int_{a}^{b} p(t) d t, \quad \text { and } \quad m \leq f(t) \leq M, \quad 0 \leq p(t), \quad \forall a \leq t \leq b,
$$


with $m$ and $M$ may be infinite, and $f(t)$ is almost always different from $m$ and $M$. Then

$$
\phi\left(\frac{\int_{a}^{b} p(t) f(t) d t}{\int_{a}^{b} p(t) d t}\right) \leq \frac{\int_{a}^{b} p(t) \phi(f(t)) d t}{\int_{a}^{b} p(t) d t} .
$$

Here equality holds if and only if $f(t)$ is a constant function on $[a, b]$.

Proof of Theorem 5. We carry out the proof in two steps.

Step 1. The non-existence of $\tau / 2$-antiperiodic solutions of (6) when $\tau>0$ is small enough.

Given $\tau>0$, suppose $x=x(t)$ is a $\tau / 2$-antiperiodic solution of (6). For $r>0$ let

$$
\psi_{x}(r)=f_{\tau}(r x)=\frac{r^{2}}{2} C_{0}+a y r^{-\alpha}-b C_{\beta} r^{-\beta},
$$

where we define

$$
C_{0}=\int_{0}^{\tau}|\dot{x}(t)|^{2} d t, \quad y=\int_{0}^{\tau} \frac{1}{|x(t)|^{\alpha}} d t, \quad C_{\beta}=\int_{0}^{\tau} \frac{1}{|x(t)|^{\beta}} d t .
$$

Because $x=x(t)$ is a $\tau / 2$-antiperiodic solution of (6), we have

$$
\left.\psi_{x}^{\prime}(r)\right|_{r=1}=\left.f_{\tau}^{\prime}(r x) \cdot x\right|_{r=1}=0 .
$$

By direct computations from (25) we obtain

$$
\begin{aligned}
\left.\psi_{x}^{\prime}(r)\right|_{r=1} & =\left.\left(C_{0} r-a \alpha y r^{-\alpha-1}+b \beta C_{\beta} r^{-\beta-1}\right)\right|_{r=1} \\
& =C_{0}-a \alpha y+b \beta C_{\beta} .
\end{aligned}
$$

Because $x=x(t)$ is $\tau / 2$-antiperiodic, it satisfies $\int_{0}^{\tau} x(t) d t=0$. Thus by applying the Wirtinger's inequality (Lemma 2.1) to each coordinate function of $x \in \mathbb{R}^{2 n}$ and then adding them up, we obtain

$$
C_{0}=\int_{0}^{\tau}|\dot{x}|^{2} d t \geq\left(\frac{2 \pi}{\tau}\right)^{2} \int_{0}^{\tau}|x|^{2} d t .
$$

Let $\phi(t)=t^{-2 / \alpha}$, then it is strictly convex, because $\phi^{\prime \prime}(t)=\frac{2(2+\alpha)}{\alpha^{2}} t^{-(2+2 \alpha) / \alpha}>0$ for all $t>0$. Then by Jensen's inequality (Lemma 8) with $\phi(t)=t^{-2 / \alpha}, a=0$, $b=\tau, p(t)=1$ and $f=1 /|x|^{\alpha}$, we obtain

$$
\int_{0}^{\tau}|x|^{2} d t \geq \tau^{(2+\alpha) / \alpha}\left(\int_{0}^{\tau} \frac{1}{|x|^{\alpha}} d t\right)^{-2 / \alpha}=\tau^{(\alpha+2) / \alpha} y^{-2 / \alpha} .
$$

Let $\phi(t)=t^{\beta / \alpha}$, then it is strictly convex, because $\phi^{\prime \prime}(t)=\frac{\beta(\beta-\alpha)}{\alpha^{2}} t^{(\beta-2 \alpha) / \alpha}>0$ for all $t>0$. Then by Jensen's inequality with $\phi(t)=t^{\beta / \alpha}, a=0, b=\tau, p(t)=1$ and $f=1 /|x|^{\alpha}$, we obtain

$$
C_{\beta}=\int_{0}^{\tau} \frac{1}{|x|^{\beta}} d t \geq \tau^{(\alpha-\beta) / \alpha}\left(\int_{0}^{\tau} \frac{1}{|x|^{\alpha}} d t\right)^{\beta / \alpha}=\tau^{(\alpha-\beta) / \alpha} y^{\beta / \alpha} .
$$

Plugging all the above estimates into (28) we obtain

$$
\left.\psi_{x}^{\prime}(r)\right|_{r=1} \geq 4 \pi^{2} \tau^{(2-\alpha) / \alpha} y^{-2 / \alpha}-a \alpha y+b \beta \tau^{-(\beta-\alpha) / \alpha} y^{\beta / \alpha} \equiv \xi(y),
$$

for some $y>0$. 
Let $\tau_{1}>0$ be sufficiently small such that $b \beta \tau^{-(\beta-\alpha) / \alpha} \geq a \alpha$ for all $\tau \in\left(0, \tau_{1}\right]$. Then for all $y \geq 1$, we obtain $b \beta \tau^{-(\beta-\alpha) / \alpha} y^{\beta / \alpha}-a \alpha y \geq 0$ and

$$
\xi(y) \geq \tau^{-(\beta-\alpha) / \alpha}>0 .
$$

Here we used the condition $0<\alpha<\beta$.

Let $\tau_{2}>0$ be sufficiently small such that $4 \pi^{2} \tau^{(2-\alpha) / \alpha} \geq a \alpha$ for all $\tau \in\left(0, \tau_{2}\right]$. Then for all $0<y<1$, we obtain $4 \pi^{2} \tau^{(2-\alpha) / \alpha} y^{-2 / \alpha}-a \alpha y \geq 0$ and

$$
\xi(y) \geq b \beta \tau^{-(\beta-\alpha) / \alpha} y^{\beta / \alpha}>0 .
$$

Here we used the condition $2<\alpha$.

Therefore for all $\tau \in\left(0, \min \left\{\tau_{1}, \tau_{2}\right\}\right)$, we obtain

$$
\left.\psi_{x}^{\prime}(r)\right|_{r=1} \geq \xi(y)>0 .
$$

Together with (27), it yields a contradiction, whenever $\tau \in\left(0, \min \left\{\tau_{1}, \tau_{2}\right\}\right)$. Therefore when $\tau>0$ is sufficiently small, system (6) possesses no $\tau / 2$-antiperiodic solutions.

Step 2. The existence of $\tau^{*}$ claimed in Theorem 5.

By Propositions 3 and 4 , for $\tau \geq \tau^{* *}$ with $\tau^{* *}$ being the unique positive root of $\chi(\tau)$ of (10), (6) possesses always $\tau / 2$-antiperiodic circular solutions. Therefore by Step 1 , there exists a $\tau^{*}>0$ such that for $\tau \in\left(0, \tau^{*}\right)$ there exist no $\tau / 2$ antiperiodic solutions of (6), and for $\tau=\tau^{*}$ the system (6) possesses at least one $\tau / 2$-antiperiodic solution.

Now other claims of Theorem 5 follow from Propositions 3 and 4 .

The proof of Theorem 5 is complete.

Remark 1. (i) By our above study, it is natural to ask whether for every $\tau \geq \tau^{*}$, the system (6) possesses any $\tau / 2$-antiperiodic solutions which are not circular motions.

(ii) It is not clear so far whether $\tau^{*}=\tau^{* *}$ holds, as well as whether there exists any $\tau / 2$-antiperiodic solutions for $\tau \in\left(\tau^{*}, \tau^{* *}\right)$ if $\tau^{*}<\tau^{* *}$ holds.

(iii) Based on results in Propositions 3 and 4 , it is natural to ask whether the Theorem 5 continues to hold when the potential function is a weak force, i.e., $0<$ $\alpha \leq 2<\beta$.

(iv) Here we would like to draw readers attentions to a remarkable result of Ambrosetti and Coti Zelati, i.e., Theorem 9.1 of [1] in 1993, in which they proved the existence of at least one $\tau$-periodic solution of the system (6) for every $\tau>0$. In their proof, they constructed a mountain pass structure which depends on a set of suitable functions with non-zero mean integral values. Their $\tau$-periodic solutions are not $\tau / 2$-antiperiodic. For $0<\tau<\tau^{*}$, this conclusion follows from our Theorem 5. Here a natural task of the future study on the system (6) is to understand the global structures of the sets of its $\tau / 2$-antiperiodic solutions and $\tau$-periodic solutions respectively for prescribed suitable $\tau>0$ with the potential function being strong force or weak force.

\section{ACKNOWLEDGEMENTS}

The first author is partially supported by the MINECO/FEDER grant MTM200803437 and MTM2013-40998-P, the Generalitat de Catalunya grant 2014SGR-568, ICREA Academia, and two grants FP7-PEOPLE-2012-IRSES 316338 and 318999 and from the recruitment program of high-end foreign experts of China. 
The second author is supported by NSFC Grant 11131004, MCME, LPMC of Ministry of Education of China, Nankai University, and the Beijing Center for Math. and Info. Inter. Sciences at Capital Normal University. He would like to express his sincere thanks to Centre de Recerca Matemàtica of the Universitat Autònoma de Barcelona for the hospitality during his visit in May 26 to June 25th of 2014. He would like to thank sincerely Professors Jaume Llibre and Ernesto Pérez-Chavela for inviting him to give a lecture in HAMSYS-2014.

\section{REFERENCES}

[1] A. Ambrosetti, V. Coti Zelati, Periodic Solutions of Singular Lagrangian Systems, Progress in Nonlinear Diff. Equa. and Their Appl. 10, Birkhäuser, Boston, 1993.

[2] M. Barbosu, V. Mioc, D. Pas,ca and F. Szenkovits, The two-body problem with generalized Lennard-Jones potential, J. Math. Chem. 49 (2011), 1961-1975.

[3] S.G. Brush, Interatomic forces and gas theory from Newton to Lennard-Jones, Arch. Rational Mech. Anal. 39 (1970), 1-29.

[4] M. Corbera, J. Llibre and E. Pérez-Chavela, Equilibrium points and central configurations for the Lennard-Jones 2- and 3-body problems, Celestial Mechanics and Dynamical Astronomy 89 (2004), 235-266.

[5] D. Frenkel and B. Smit, Understanding Molecular Simulation, 2nd edn., Academic Press, San Diago, 2002.

[6] G. Hardy, J. Littlewood and G. Polya, Inequalities, 2nd ed, University Press, Cambridge. 1952.

[7] E. Lacomba and J. Llibre, Integrals, invariant manifolds and degeneracy for central force problems in $\mathbb{R}^{n}$, J. Mathematical Physics 33 (1992), 2138-2147.

[8] J.E. Lennard-Jones, Cohesion, Proc. Phys. Soc. 43 (1931), 461-482.

[9] E.G. Lewars, Computational Chemistry: Introduction to the Theory and Applications of Molecular and Quantum Mechanics, Springer, Berlin, 2003.

[10] J. Llibre and C. Valls, On the $C^{1}$ non-integrability of differential systems via periodic orbits, European J. of Applied Mathematics 22 (2011), 381-391.

[11] Y. Long and S. Zhang, Geometric characterizations for variational minimization solutions of the 3-body problem, Acta Math.Sinica. 16 (2000), 579-592.

[12] K.R. Meyer, G,R. Hall and D. Offin, Introduction to Hamiltonian dynamical systems and the $N$-body problem, second edition, Applied Mathematical Sciences 90, Springer, New York, 2009.

[13] D. Pasca and C. Valls, Qualitative analysis of the anisotropic two-body problem with generalized Lennard-Jones potential, J. Math. Chem. 50 (2012), 2671-2688.

[14] L. Sbanoa and J. Southall, Periodic solutions of the N-body problem with Lennard-Jonestype potentials, Dynamical Systems 25 (2010), 53-73.

1 Departament de Matemàtiques, Universitat Autònoma de Barcelona, 08193 Bellaterra, Barcelona, Catalonia, Spain

E-mail address: jllibre@mat.uab.cat

2 Chern Institute of Mathematics and LPMC, Nankai University, Tianjin, 300071, CHINA

E-mail address: longym@nankai.edu.cn 